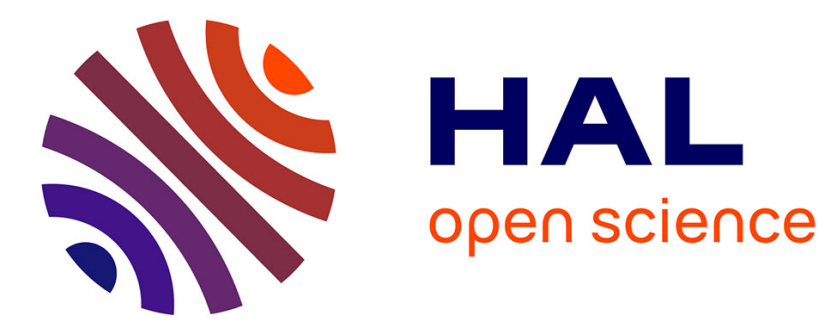

\title{
Eddy current in a rotating cylinder in a static field by a stochastic method
}

\author{
Jean Lévêque, T. Lubin, S. Mezani, A. Rezzoug
}

\section{To cite this version:}

Jean Lévêque, T. Lubin, S. Mezani, A. Rezzoug. Eddy current in a rotating cylinder in a static field by a stochastic method. European Physical Journal: Applied Physics, 2012, 57 (3), 10.1051/epjap/2011110025 . hal-00788686

\section{HAL Id: hal-00788686 https://hal.science/hal-00788686}

Submitted on 15 Feb 2013

HAL is a multi-disciplinary open access archive for the deposit and dissemination of scientific research documents, whether they are published or not. The documents may come from teaching and research institutions in France or abroad, or from public or private research centers.
L'archive ouverte pluridisciplinaire HAL, est destinée au dépôt et à la diffusion de documents scientifiques de niveau recherche, publiés ou non, émanant des établissements d'enseignement et de recherche français ou étrangers, des laboratoires publics ou privés. 


\title{
Eddy current in a rotating cylinder in a static field by a stochastic method
}

\author{
J. Lévêque, T. Lubin, S. Mezani, A. Rezzoug \\ GREEN University of Nancy BP 23954506 Vandoeuvre France
}

\begin{abstract}
This paper deals with the calculation of eddy current in copper cylinder. This cylinder rotates in an imposed static magnetic field. The electromagnetic problem is solved in two-dimension by considering transient motion. Two methods for eddy current computation are compared: stochastic method and classical Finite Element Method. The main goal of this paper is to compare these methods.
\end{abstract}

\section{Introduction}

In this paper we present the calculation of eddy current in a copper cylinder when it moves in an applied static magnetic field. This is a very important problem for thermal treatment of metal [1-2]. For heating any metal by induction field, we have two solutions. The first one is to submit the conductor material to a low value (about $0.7 \mathrm{~T}$ ) and high frequency variable magnetic field. The second method consists in the achievement of a high magnetic field (several Tesla), by using a superconducting magnet for example, in which the material can rotate. So, the control of the body temperature is obtained by adjusting the rotating speed and/or the value of the magnetic field. There are some studies on this kind of heating. In this paper we propose a new numerical method to calculate eddy current distribution in the cylinder. The aim of this paper is to present a stochastic method to calculate induced current in a moving material. This method has some advantage like calculation in only one point or in some points and not in the whole domain. We present these advantages on a $2 \mathrm{D}$ transient motion problem with a constant applied magnetic field. The results obtained with the proposed method are compared with those obtained by the classical finite element method.

\subsection{Equation to be solve}

First, we present the equation to solve [3] in order to find the induced current in a cylinder which rotates in a uniform magnetic field. The considered problem is solved in twodimension (2D). From this assumption, the flux density presents only two components in the $x-y$ plane and the potential vector has only one component, this one being orthogonal to this plane ( $z$-component).

The induced current density in the cylinder is given by

$$
\mathrm{J}=\sigma_{e}(\mathrm{e}+\mathrm{v} \times \mathrm{B})
$$

where $\sigma_{\mathrm{e}}$ is the electrical conductivity of the cylinder.
The relation between the magnetic vector potential and the current density is

$$
\Delta \mathbf{A}=-\mu_{0} \mathbf{J}
$$

Substituting (1) into (2), we obtain

$$
\Delta \mathrm{A}=-\mu_{0} \sigma_{e}(\mathrm{e}+\mathrm{v} \times \mathrm{B})
$$

The relation between the electric field and the magnetic vector potential is defined as:

$\mathbf{e}=-\frac{\partial \mathbf{A}}{\partial t}-\operatorname{grad}(V)$

Equation (4) is then replaced in (3) to give:

$$
\Delta \mathrm{A}=+\mu_{0} \sigma_{e} \frac{\partial \mathrm{A}}{\partial t}-\mu_{0} \sigma_{e}(\mathrm{v} \times \mathrm{B})
$$

Now, we have to calculate the term $(\mathrm{v} \times \mathrm{B})$. To do that, we define the position of a point $\mathrm{M}$ and the components of the vector speed in cylindrical coordinates system:

$$
\begin{aligned}
& \overrightarrow{\mathrm{OM}}=\begin{array}{l}
\mathrm{r} \cos \theta \\
\mathrm{r} \sin \theta \\
0
\end{array} \\
& \mathbf{v}=\mid \begin{array}{l}
-\mathrm{r} \dot{\theta} \sin \theta=\dot{\theta} \mathrm{y} \\
\mathrm{r} \dot{\theta} \cos \theta=\dot{\theta} \mathrm{x} \\
0
\end{array}
\end{aligned}
$$

$\dot{\theta}$ or $(\mathrm{d} \theta / \mathrm{dt})$ is the angular speed of the copper cylinder

Knowing that the magnetic field is given by:

$$
\mathrm{B}=\operatorname{curl}(\mathrm{A})=\mid \begin{aligned}
& \partial \mathrm{A} / \partial y \\
& -\partial \mathrm{A} / \partial x \\
& 0
\end{aligned}
$$


The product $(\mathrm{v} \times \mathrm{B})$ is developed and gives:

$$
\mathrm{v} \times \mathrm{B}=\mid \begin{aligned}
& 0 \\
& 0 \\
& \partial \mathrm{A} / \partial x r \dot{\theta} \sin \theta-\partial \mathrm{A} / \partial y r \dot{\theta} \cos \theta
\end{aligned}
$$

We now have to solve the following equation in the cylinder and in the air regions:

$$
\Delta \mathrm{A}=+\mu_{0} \sigma_{e} \frac{\partial \mathrm{A}}{\partial t}+\mu_{0} \sigma_{e}(-\partial \mathrm{A} / \partial x y \dot{\theta}+\partial \mathrm{A} / \partial y x \dot{\theta})
$$

where $\sigma=0$ in the air region.

\subsection{Boundary condition}

Now, we need to set the boundary conditions. The rotating cylinder is placed in a constant magnetic field, as shown in figure $n^{\circ} 1$.

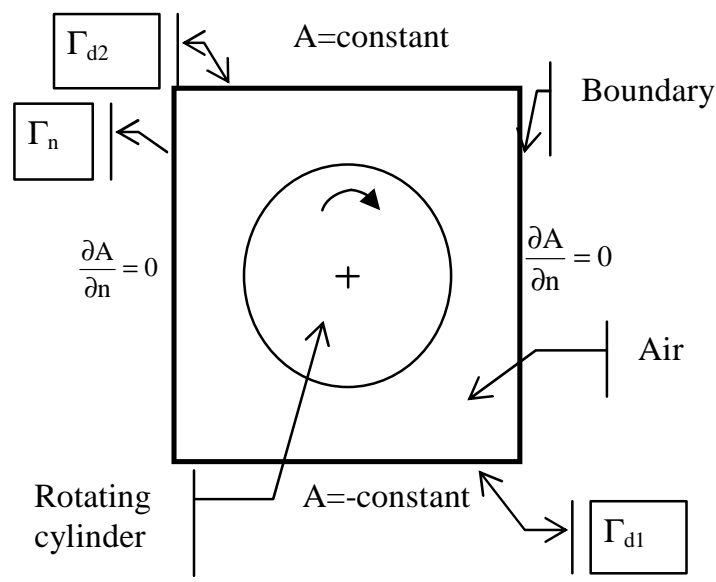

Fig. 1. Rotating cylinder in a constant magnetic field

The whole considered domain, air and rotating cylinder regions, is a square. On two sides of the domain, we have Neumann's condition and on the other sides, the value of the magnetic vector potential is imposed, either a constant or its opposite. With these boundary conditions, we have a constant and uniform magnetic field created by external sources. In the study, the boundaries are placed far enough from the copper cylinder to not perturb the solution.

So, we now have the following problem to solve:

$$
\left\{\begin{array}{l}
\Delta \mathrm{A}=+\mu_{0} \sigma_{e} \frac{\partial \mathrm{A}}{\partial t}+\mu_{0} \sigma_{e}(\partial \mathrm{A} / \partial x y \dot{\theta}+\partial \mathrm{A} / \partial y x \dot{\theta}) \\
\text { with } \sigma=0 \text { in air } \\
\text { and } \\
\frac{\partial \mathrm{A}}{\partial \mathrm{n}}=0 \text { in } \Gamma_{n} \\
\mathrm{~A}=- \text { Cte in } \Gamma_{d 1} \\
\mathrm{~A}=+ \text { Cte in } \Gamma_{d 2}
\end{array}\right.
$$

We have also a condition on the movement of the cylinder. The speed increase linearly with time from 0 to $3 \mathrm{rd} . \mathrm{s}^{-1}$ during $100 \mathrm{~ms}$. So, the initial value of the speed is null.

In this first part, the system of equations to describe the problem has been developed. In the two following parts, we present first the stochastic method allowing to solve the problem and then the obtained results.

\section{Calculation by stochastic method}

Stochastic methods are used to solve various kinds of problem but are very rarely used in electrical engineering. To show the relevance of such method, we applied it to solve our problem [4-7].

\subsection{Stochastic resolution of PDE}

The aim of this part is to explain how to solve equation (11). We begin by a short presentation of Monte-Carlo method to describe the resolution of PDE by stochastic process. In a second step, we present the resolution of our problem thanks to the Brownian motion.

\subsubsection{Fixed random walk}

The resolution of a stationary problem by a Monte Carlo method consists in generating a set of fixed random walks starting from a point of calculation. A virtual particle randomly moves on a mesh. A random walk stops when the particle reaches a border with a Dirichlet's condition. The particle retrogresses when it meets a border with a Newman's condition. The probabilities of displacement are given by the discretization of the partial derivative equation as explain in the following example.

We will generalise the construction of a random walk to solve a time dependent problem. We take for example a very simple one-dimensional diffusion equation:

$$
\frac{\partial U}{\partial t}=\frac{\partial^{2} U}{\partial x^{2}}
$$

The first step consists of discretizing the PDE. We notice that $\mathrm{x}=\mathrm{i} \cdot \Delta \mathrm{x}$ and $\mathrm{t}=\mathrm{n} . \Delta \mathrm{t}$, where $\Delta \mathrm{x}$ and $\Delta \mathrm{t}$ are the spatial and temporal steps.

$$
\frac{\partial^{2} \mathrm{U}}{\partial \mathrm{x}^{2}} \approx \frac{\mathrm{U}_{\mathrm{i}+1, \mathrm{n}}-2 \mathrm{U}_{\mathrm{i}, \mathrm{n}}+\mathrm{U}_{\mathrm{i}-1, \mathrm{n}}}{\Delta \mathrm{x}^{2}}
$$

and

$$
\frac{\partial \mathrm{u}}{\partial \mathrm{t}} \approx \frac{\mathrm{U}_{\mathrm{i}, \mathrm{n}}-\mathrm{U}_{\mathrm{i}, \mathrm{n}-1}}{\Delta \mathrm{t}}
$$


Substituting (13), (14) and (15) into (12), we obtain the following equation

$$
\begin{aligned}
& U_{i+1, n}-2 U_{i, n}+U_{i-1, n}=\alpha\left(U_{i, n}-U_{i, n-1}\right) \\
& \mathrm{u}_{\mathrm{i}, \mathrm{n}}=\frac{\mathrm{U}_{\mathrm{i}+1, \mathrm{n}}}{2+\alpha}+\frac{\mathrm{U}_{\mathrm{i}-1, \mathrm{n}}}{2+\alpha}+\frac{\alpha \cdot \mathrm{U}_{\mathrm{i}, \mathrm{n}-1}}{2+\alpha}
\end{aligned}
$$

with

$$
\alpha=\frac{\Delta \mathrm{x}^{2}}{\Delta \mathrm{t}}
$$

From this equation, we can define the following probabilities:

$$
\begin{aligned}
& \mathrm{P}_{\mathrm{x}+}=\mathrm{P}_{\mathrm{x}-}=\frac{1}{2+\alpha} \\
& P_{t-}=\frac{\alpha}{2+\alpha}
\end{aligned}
$$

Thus, we have: $\mathrm{p}_{\mathrm{x}-}+\mathrm{p}_{\mathrm{x}+}+\mathrm{p}_{\mathrm{t}}=1$.

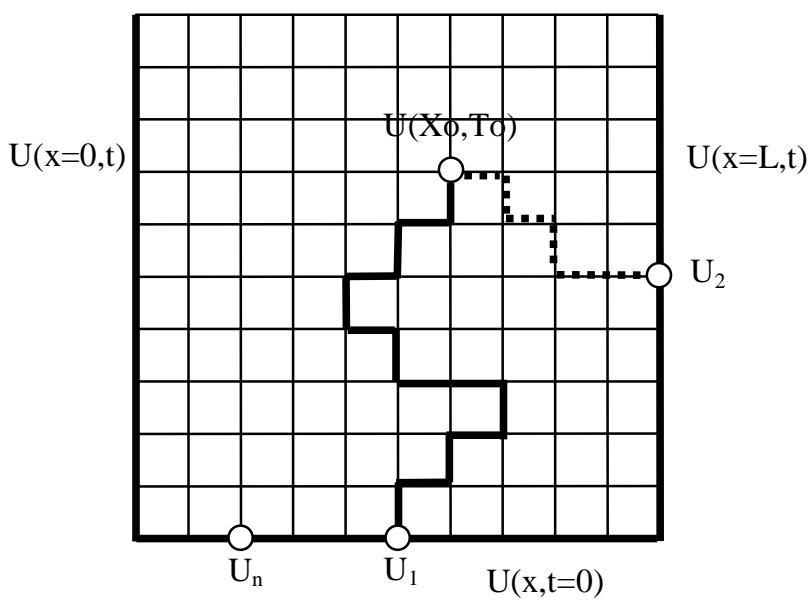

Fig. 2. Spatio temporal random walk. $U(X o, T o)=\frac{1}{N} \sum_{n=1}^{N} U_{n}$

So, we define the probabilities which will be used to build the random walks.

$\mathrm{U}_{\mathrm{i}, \mathrm{n}}=\mathrm{p}_{\mathrm{x}-} \mathrm{U}_{\mathrm{i}-1, \mathrm{n}}+\mathrm{p}_{\mathrm{x}+} \mathrm{U}_{\mathrm{i}+1, \mathrm{n}}+\mathrm{p}_{\mathrm{t}} \mathrm{U}_{\mathrm{i}, \mathrm{n}-1}$,

As shown in Fig. 2, the Monte Carlo Markov Chain Method consists in computing a set of random walks starting from the same point $\mathrm{P}\left(\mathrm{Xo}=\mathrm{i}_{0} . \Delta \mathrm{x}, \mathrm{To}_{\mathrm{O}} \mathrm{n}_{\mathrm{o}} . \Delta \mathrm{t}\right)$ for which we need to approximate the value of $\mathrm{U}$.

We consider that $\mathrm{dx}$ and $\mathrm{dt}$ values are quite chosen for the numerical calculation. To do that let us consider the value of $\alpha$. If it is very small, whereas the value of the probability $P_{x+}$ and $\mathrm{P}_{\mathrm{x} \text { - }}$ tends to $0.5, \mathrm{P}_{\mathrm{t}}$ tends to 0 and the convergence process is time consuming. If $\alpha$ is very large, the space probabilities tend toward 0 while $\mathrm{P}_{\mathrm{t}}$ tends to 1 . A reasonable choice for the value of $\alpha$ is roughly 1 which gives an equiprobability for the three walks.

\subsubsection{Brownian motion}

We saw that the probability of displacement on the mesh comes from the discretization by finite difference of the equation (12). Knowing that when the spatial step tends to zero, the random walk tends to a Brownian motion, we use this property to build now a random walk without mesh.

The general expression of a diffusion processes is given by:

$\frac{\partial U}{\partial t}=L u(x, t)=\frac{1}{2} \sum_{i, j=1}^{d} S_{i j}(x) \frac{\partial^{2} U}{\partial x_{i} \partial x_{j}}+\sum_{i=1}^{d} \mu_{i}(x) \frac{\partial U}{\partial x_{i}}$

where $x_{i}, x_{j}$ are spatial variable and the time.

It could be associated to a diffusion process which is solution of the Cauchy problem [8]:

$d X(t)=\mu(t, X(t)) d t+\sigma(t, X(t)) d B_{t}$

$\mathrm{S}_{\mathrm{ij}}(\mathrm{x})$ being a square matrix, we have the following relation:

$\mathrm{S}_{\mathrm{ij}}(\mathrm{x})=\sigma^{\mathrm{T}}(\mathrm{x}) \cdot \sigma(\mathrm{x})$

$\mathrm{X}(\mathrm{t})$ is a stochastic variable which defines the position of a particle at a time $t$. At $t=0$ this particle is at the position where we need to calculate the solution of the equation.

$\mu(t, X(t))_{t}$ is the expectation of the stochastic variable.

$\sigma^{2}(t, X(t))$ is the variance of the stochastic variable.

$\mathrm{B}_{\mathrm{t}}$ is the Brownian motion. It is a stochastic variable whose increment $B_{t+\delta t}-B_{t}$ follows a normal distribution law $N(0, \delta t)$.

With this method, a stationary problem is calculated like the limit of a time dependent problem.

We now present a simple example to illustrate the calculation process. We consider the Laplace equation in $2 \mathrm{D}$ :

$\alpha \frac{\partial^{2} U}{\partial x^{2}}+\beta \frac{\partial^{2} U}{\partial y^{2}}=0$

This equation could be rewritten to correspond to the form defined by (21):

$\frac{1}{2}\left(2 \alpha \frac{\partial^{2} \mathrm{U}}{\partial \mathrm{x}^{2}}+2 \beta \frac{\partial^{2} \mathrm{U}}{\partial \mathrm{y}^{2}}\right)=0$

The parameters of the stochastic variable $\mathrm{X}(\mathrm{t})$ are now the following : 
$\mathrm{S}_{\mathrm{ij}}(\mathrm{x})=\left(\begin{array}{cc}2 \alpha & 0 \\ 0 & 2 \beta\end{array}\right)=\sigma^{\mathrm{T}} \sigma$

where

$\sigma=\left(\begin{array}{cc}\sqrt{2 \alpha} & 0 \\ 0 & \sqrt{2 \beta}\end{array}\right)$

and $\mu=0$.

\subsubsection{How many Brownian motion do we have to take?}

It is obvious that we cannot take only one Brownian motion to estimate the solution at the point $\mathrm{P}\left(\mathrm{X}_{0}, \mathrm{~T}_{\mathrm{o}}\right)$. The question is how many Brownian motion we have to take?

Let us define the stochastic variable $\mathrm{U}_{\mathrm{N}}$ by:

$\mathrm{U}_{\mathrm{N}}=\frac{1}{\mathrm{~N}} \sum_{\mathrm{n}=1}^{\mathrm{n}=\mathrm{N}} \mathrm{U}_{\mathrm{n}}$

The stochastic variable associated to the $\mathrm{n}^{\text {th }}$ Brownian displacement is $U_{n} \cdot \tilde{U}_{n}$ is the simulation result for the $n^{\text {th }}$ random process starting from the point $\mathrm{P}(\mathrm{xo}, \mathrm{to})$. The central limit theorem indicates that $\mathrm{U}_{\mathrm{N}}$ follows a Gaussian stochastic law with $\mathrm{U}_{\mathrm{S}}$ the mathematical expectation and with $\sigma_{S} / \sqrt{N}$ standard deviation if $\mathrm{N}$ is sufficiently high.

The probability that $\tilde{\mathrm{U}}_{\mathrm{n}}$ belongs to the interval $\left.\mid \mathrm{U}_{\mathrm{S}}-2 . \sigma_{\mathrm{S}} / \sqrt{\mathrm{N}}, \mathrm{U}_{\mathrm{S}}+2 . \sigma_{\mathrm{S}} / \sqrt{\mathrm{N}}\right\rfloor$ is $95 \% . \mathrm{U}_{\mathrm{S}}$ and $\sigma_{s} / \sqrt{N}$ are estimated by $\tilde{\mathrm{U}}_{\mathrm{S}}$ and $\tilde{\sigma}_{S}$ which are defined by:

$\left\{\begin{array}{l}\mathrm{S}_{1}=\sum_{\mathrm{n}=1}^{\mathrm{n}=\mathrm{N}} \tilde{\mathrm{U} n} \\ \mathrm{~S}_{2}=\sum_{\mathrm{n}=1}^{\mathrm{n}=\mathrm{N}} \tilde{\mathrm{U}}^{2}\end{array}\right.$ and $\left\{\begin{array}{l}\tilde{\mathrm{U}}_{\mathrm{S}}=\frac{\mathrm{S}_{1}}{\mathrm{~N}} \\ \tilde{\mathrm{\sigma}}_{\mathrm{S}}=\frac{1}{\mathrm{~N}-1} \sqrt{\mathrm{S}_{2}-\frac{\mathrm{S}_{1}{ }^{2}}{\mathrm{~N}}}\end{array}\right.$

Where subscript $\mathrm{n}$ correspond to the current Brownian motion and subscript $\mathrm{s}$ correspond to the final value of the stochastic variable

As $\mathrm{N}$ increases, the diameter of the $95 \%$ confidence interval $\left\lfloor\mathrm{U}_{\mathrm{S}}-2 . \sigma_{\mathrm{S}} / \sqrt{\mathrm{N}}, \mathrm{U}_{\mathrm{S}}+2 . \sigma_{\mathrm{S}} / \sqrt{\mathrm{N}}\right\rfloor$ tends to zero and $\tilde{\mathrm{U}}_{\mathrm{S}}$ tends to the solution $\mathrm{U}_{\mathrm{S}}$.

We obtain not only one estimate of the solution but also an estimate of the error by the value of the standard deviation $\tilde{\sigma}_{S}$.

\subsection{Application to a rotating cylinder}

In this part, we use Brownian motion to solve the problem of a copper cylinder rotating in magnetic field as represented in figure 1. To solve equation (11), we first rewrite it in order to separate time derivative term:

$\mu_{0} \sigma_{e} \frac{\partial \mathrm{A}}{\partial t}=\Delta \mathrm{A}-\mu_{0} \sigma_{e}(\partial \mathrm{A} / \partial x y \dot{\theta}+\partial \mathrm{A} / \partial y x \dot{\theta})$

$\frac{\partial A}{\partial t}=\frac{1}{\mu_{0} \sigma}\left\{\frac{\partial^{2} A}{\partial x^{2}}+\frac{\partial^{2} A}{\partial y^{2}}\right\}-\dot{\theta}\left\{y \frac{\partial A}{\partial x}+x \frac{\partial A}{\partial y}\right\}$

An elementary displacement of the Brownian motion is given by the previously described equation:

$d X(t)=\mu(t, X(t)) d t+\sigma(t, X(t)) d B_{t}$

In this case, the parameters are the following characteristics:

$\sigma(t, X(t))=\left[\begin{array}{cc}\sqrt{\frac{2}{\mu_{0} \sigma_{e}}} & 0 \\ 0 & \sqrt{\frac{2}{\mu_{0} \sigma_{e}}}\end{array}\right]$

and

$\mu(t, X(t))=-\dot{\theta}\left\{\begin{array}{l}y \\ x\end{array}\right\}$

The main characteristics of the studied problems are summarized in table 1

Table 1. Characteristic of the problem

\begin{tabular}{lll}
\hline & Copper & Air \\
\hline $\begin{array}{l}\text { Angular speed } \\
\text { Electrical } \\
\text { conductivity }\end{array}$ & 0 to $3 \mathrm{rd} / \mathrm{s}$ & 0 \\
Size & $3710^{6} \mathrm{~S} / \mathrm{m}$ & 0 \\
& & \\
Step size & cylinder radius of & Square of $0.16 \mathrm{~m}$ \\
Simulation time & $0.08 \mathrm{~m}$ & \\
Time step & & $0.004 \mathrm{~m}$ \\
\hline
\end{tabular}

The value of the magnetic vector potential is fixed at 0.1 $\mathrm{Wb} / \mathrm{m}$ on the boundary $\Gamma_{\mathrm{d} 2}$ and at $-0.1 \mathrm{~Wb} / \mathrm{m}$ on the boundary $\Gamma_{\mathrm{d} 1}$. These values give a constant magnetic flux density of 1.25 $\mathrm{T}$ in the whole domain when the copper cylinder doesn't move.

Electrical conductivity $\sigma_{\mathrm{e}}$ and angular speed are null in the air region and have a non null value in the copper cylinder region. To overcome the numerical problem due to any discontinuity of the parameters in equation (11), functions $\sigma(t, X(t))$ and $\mu(t, X(t))$ must be continuous. For that, we adopt a sigmoid representation of speed and conductivity 


$$
\begin{aligned}
& \sigma_{\mathrm{e}}(\mathrm{x})=\sigma_{\mathrm{e}} \mathrm{e}^{\left(\left(\mathrm{x}^{2}+\mathrm{y}^{2}\right) / \mathrm{c}\right)^{\mathrm{d}}} \\
& \dot{\theta}(\mathrm{x})=\dot{\theta} \mathrm{e}^{\left(\left(\mathrm{x}^{2}+\mathrm{y}^{2}\right) / \mathrm{c}\right)^{\mathrm{d}}},
\end{aligned}
$$

where $\mathrm{c}$ and $\mathrm{d}$ are parameters adjusting the curves. The variation of the electrical conductivity versus space is shown on figure 3. If the c term is too weak, the variation of the speed is not sharp and the description of the phenomenon is not good. We do not see influence of this term on the computation speed and the precision of the calculus.

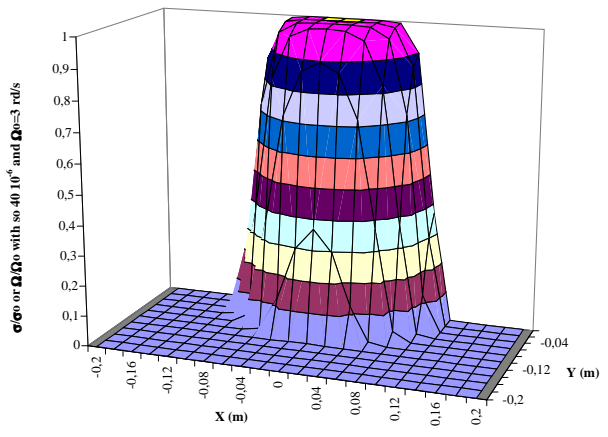

Fig. 3. Electrical conductivity versus space for $c=0.01$ and $\mathrm{d}=5.3$

\section{Results}

In order to study the effectiveness of the stochastic method, the results have been compared with those obtained from $2 \mathrm{D}$ transient finite element simulations.

Figure 4 and figure 5 show the flux lines obtained respectively by stochastic method and finite element simulation for the same condition. We can observe that the results are in good agreement but very noisy with stochastic method.

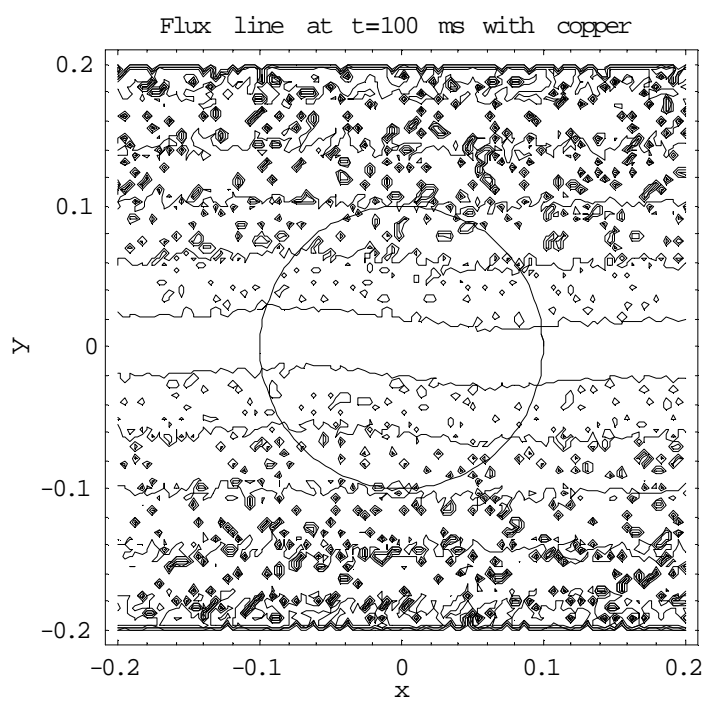

Fig. 4. Flux lines obtained with Brownian motion method

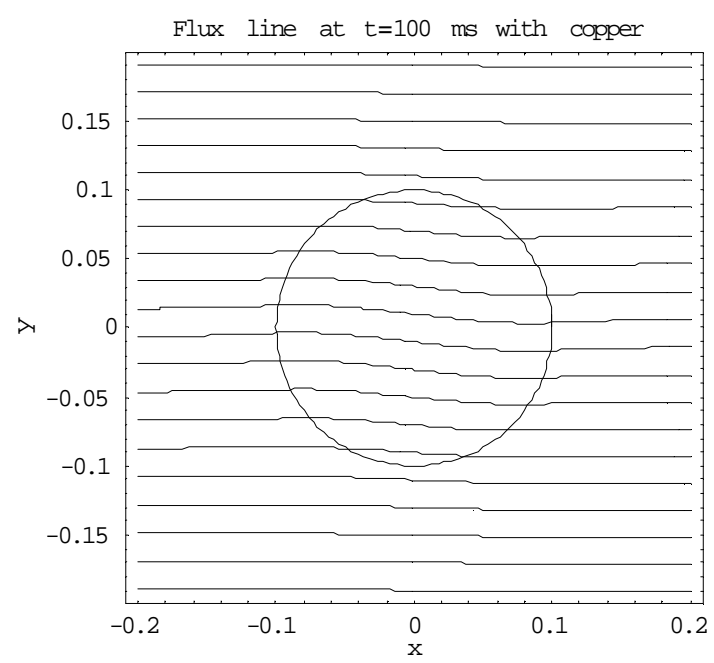

Fig. 5. Flux lines obtained with finite element simulation.

Figure 6 shows the time evolution of the potential vector at a given point $(\mathrm{x}=0.8, \mathrm{y}=0.8)$ on the cylinder surface obtained by finite element simulation and Brownian motion. We note again a good agreement between the results obtained by the two methods.

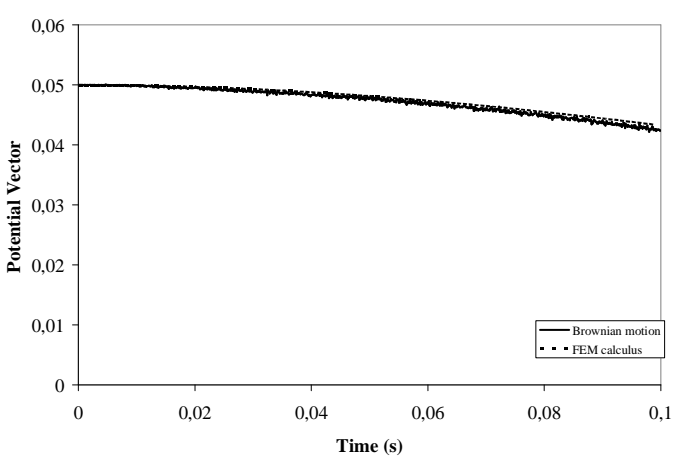

Fig. 6. Comparison of the two methods at one point on the border of the cylinder

The main advantage of the stochastic method is in the choice of the number of Brownian motions and consequently in the precision of the calculus because we have an estimator of the results. If we need very short computer time consuming for the calculus, we can associate the stochastic method with a numeric filter to avoid the noisy part of the numerical results. Figure 7 and 8 show a comparison between two computations results of the magnetic vector potential, one with a low number of Brownian motions and another with finite element method. We also present on Fig. 7 the curve obtained thanks to a noise filtering. It can be seen in Fig. 7 and 8 that the results are much closed for FEM and stochastic method. We show on Fig. 9 a comparison between stochastic method and FEM on a line at $y=0.04$ and we a good agreement between the two methods. 


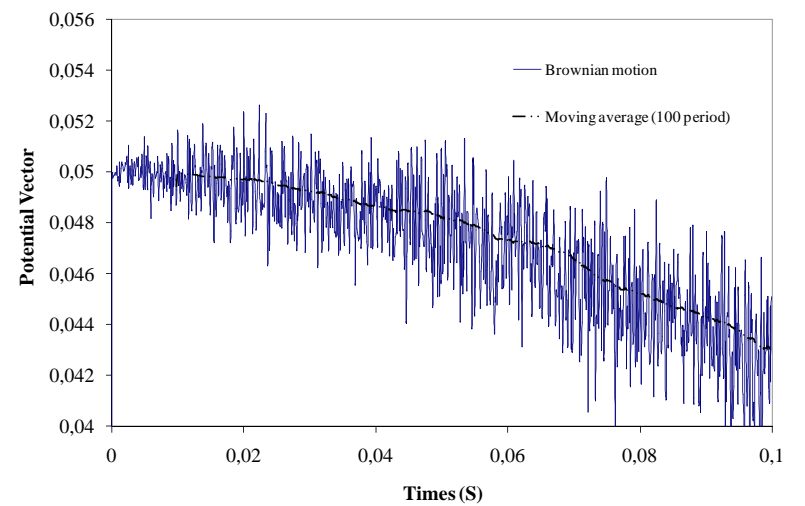

Fig. 7. Comparison of two stochastic methods at one point on the border of the cylinder

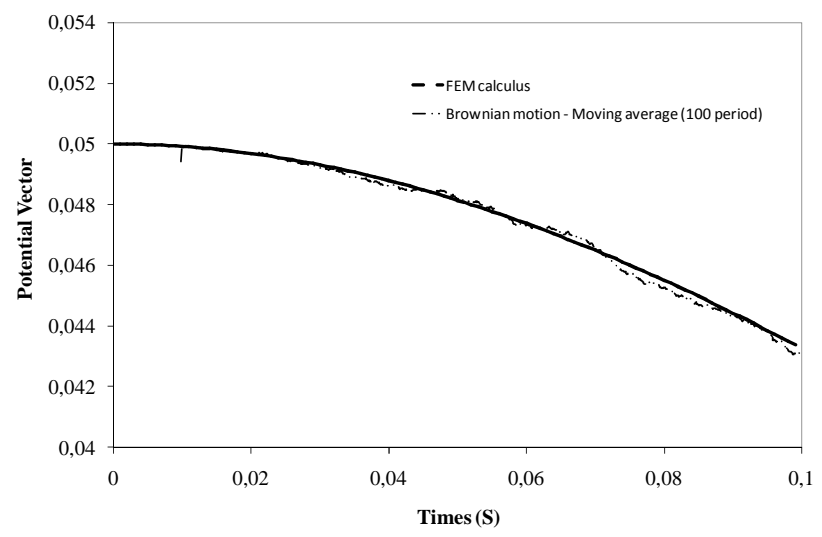

Fig. 8. Comparison of stochastic methods and finite element method at one point on the border of the cylinder

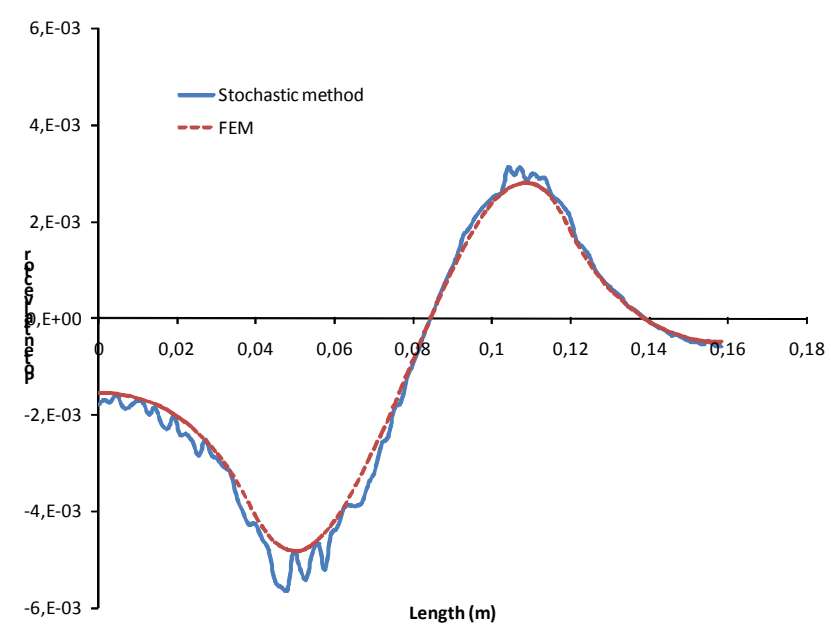

Fig. 9. Comparison of stochastic methods and finite element method on a line at $\mathrm{y}=0.04$.

\section{Conclusion}

In this paper, we have solved the problem of induced current in a moving copper cylinder placed in a uniform magnetic field. We have shown that the stochastic method gives results very close to those obtained by finite element simulations. Each method has its own advantages and drawbacks.

Among the advantages of the finite element method, we can quote its popularity and the large number of software packages, some of them being free. One of the problems of this method is that we need to calculate the solution in the whole space and for transient problem, at each space time. Another problem is that we have, when thin meshing is required, a large system to solve with the risk of numerical instability.

Among the advantages of stochastic method to solve partial differential equation, we can notice that:

- It is very easy to program it.

- It is not necessary to mesh all space and, so, to use a lot of computer memory.

- It is possible to calculate solution in only one point and not in the whole space.

- It is also possible to calculate solution at one time and not at each space time.

Because of these advantages, the method can be easily included in a larger design program to study problems of eddy currents and new thermal heating systems.

\section{References}

[1] T. Lubin, D. Netter, J. Leveque, A. Rezzoug, IEEE Transactions on Magnetics, vol.45, no.5, pt.1, May 2009, pp. 2118-27.

[2] T. Watanabe, T. Todaka, M. Enokizono, IEEE Transactions on Magnetics, vol.41, no.5, pt.1, May 2005, pp. 1884-87.

[3] M. Fabbri, M. Forzan, S. Lupi, A. Morandi, P. L. Ribani, IEEE Transactions on Magnetics, vol.45, no.5, pt.1, January 2009, pp. 192-200.

[4] K.R.Davey, M.B.Nair, IEEE Transactions on Magnetics, vol 29, n², March 1993, pp. 1376-9.

[5] K.R. Davey, , The International Journal for Computation and Mathematics in Electrical and Electronic Engineering, 2005, vol.24, no.1, pp. 229-37,

[6] K.R.Davey, Proc. COMPUMAG 2003, Saratoga Springs, July 2003.

[7] D. Netter, J. Lévêque, P. Masson, A. Rezzoug, Proc. EUCAS 2003, Sorrente, septembre 2003.

[8] P.E. Kloeden, E. Platen, "Numerical solution of stochastic differential equations", Springer-Verlag, New-York, 1992 